\title{
El audiovisual Tutankamon como herramienta para la evaluación de la competencia transversal de pensamiento crítico y responsabilidad ética, medioambiental y profesional. Una experiencia en la Universitat Politècnica de València
}

\begin{abstract}
Francisca Ramón Fernández ${ }^{a}$, Vicente Cabedo Mallol ${ }^{b}$, María Emilia Casar Furióc, Vicent Giménez Chornet ${ }^{\mathrm{d}}$, Cristina Lull Noguera ${ }^{\mathrm{e}}$, Juan Vicente Oltra Gutiérrez ${ }^{\mathrm{f}}$ y Enrique Orduña-Malea ${ }^{\mathrm{g}}$

aProfesora titular. Departamento de Urbanismo, Universitat Politècnica de València, frarafer@urb.upv.es, ${ }^{b}$ Profesor contratado doctor, acreditado a titular. Departamento de Urbanismo, Universitat Politècnica de València, vicamal@urb.upv.es, 'Profesora contratada doctora, acreditada a titular. Departamento de Urbanismo, Universitat Politècnica de València, macafu@urb.upv.es, ${ }^{\mathrm{d}}$ Profesor titular. Departamento de Comunicación Audiovisual, Documentación e Historia del Arte, Universitat Politècnica de València, vigicho@har.upv.es ${ }^{\mathrm{e}}$ Profesora contratada doctora, Departamento de Química, Universitat Politècnica de València, clull@upvnet.upv.es, ${ }^{\mathrm{f}}$ Profesor titular de Escuela Universitaria. Departamento de Organización de Empresas, Universitat Politècnica de València, jvoltra@omp.upv.es, ${ }^{\mathrm{g}}$ Profesor Ayudante Doctor. Departamento de Comunicación Audiovisual, Documentación e Historia del Arte, enorma@upv.es
\end{abstract}

\begin{abstract}
This work presents the results obtained from a new educational innovation activity applied to two Master Degrees offered at the Universitat Politècnica de València, which consisted in using one same audio-visual (Tutankamon) to evaluate - on the student side - whether students could observe two different transversal skill qualifications: a) critical thinking, and b) ethical, environmental and professional responsibility. The conclusions we have come to is that both transversal skill qualifications have been perceived by students after the episode viewing, although with differences according to the skill and Master Degree. Additionally, a high rate of acceptance of the audiovisual in the classroom has been obtained.
\end{abstract}

Keywords:

Transversal skills, critical thinking, ethical, environmental and professional responsibility, audiovisual, evaluation, Tutankamon, Master degree

\section{Resumen}

Se presentan los resultados obtenidos de una nueva actividad de innovación docente que se ha aplicado a dos titulaciones de Máster impartidas en la Universitat Politècnica de València, consistente en utilizar un mismo audiovisual, Tutankamon, para evaluar si los estudiantes eran capaces de observar dos competencias transversales distintas: pensamiento crítico y 
responsabilidad ética, medioambiental y profesional. Las conclusiones a las que hemos llegado nos muestran que las dos competencias transversales han podido ser percibidas tras el visionado del episodio, aunque con diferencias en función de la competencia y el Master. Adicionalmente, se constata un alto índice de aceptación del audiovisual en el aula.

Palabras clave: Competencias transversales, pensamiento crítico, responsabilidad ética, medioambiental y profesional, evaluación, Tutankamon, nivel Máster

\section{Introducción}

La concesión de distintos Proyectos de Innovación y Mejora Educativa (PIME) al grupo de innovación RETAJUDOCA (Recursos tecnológicos para el aprendizaje jurídico, la documentación y la comunicación audiovisual) de la Universitat Politècnica de València, en los que se aplicaron distintos elementos audiovisuales (series de animación, ficción y documentales) para la creación de un repositorio de casos prácticos y poderlos así utilizar para evaluar las competencias transversales (principalmente "pensamiento crítico" y "responsabilidad ética, medioambiental y profesional"), han supuesto la posibilidad de realizar una nueva actividad de innovación docente pionera precisamente por considerar que la utilización del audiovisual puede resultar interesante y útil para el alumnado, superando de este modo el método tradicional del caso práctico.

Por ese motivo, se va a exponer en este trabajo una nueva experiencia desarrollada durante el presente curso académico (2017-2018), que ha consistido en utilizar el audiovisual Tutankamon en dos titulaciones diferentes (Master de Gestión Cultural y Máster en Conservación y restauración de bienes culturales), con el propósito de evaluar dos competencias transversales distintas.

\section{Objetivos}

Se ha pretendido utilizar un audiovisual de ficción, en este caso un episodio de Tutankamon para poder evaluar dos competencias: CT-07 Responsabilidad ética, medioambiental y profesional y CT-09 Pensamiento crítico, en dos asignaturas diferentes ("Marco jurídico aplicado a la protección del patrimonio y a los museos" y "Legislación del Patrimonio Cultural y creación de pequeñas empresas", de forma que podamos contrastar los resultados en los alumnos, respecto a su comprensión de dichas competencias, y determinar si el audiovisual utilizado es adecuado para ello. (UPV, 2016, y Villa y Poblete, M., 2007).

Por tanto, se persiguen los siguientes objetivos principales:

a) Utilizar un audiovisual que soporta un caso práctico elaborado y publicado por los miembros del grupo de innovación docente RETAJUDOCA, dentro del marco de los PIMEs desarrollados por el grupo. Dicha publicación se ha utilizado en diversas asignaturas para evaluar los resultados a través de la resolución de un caso práctico basado en audiovisuales. En este caso, el material elegido ha sido Tutankamon (Cabedo, Casar, Giménez, Lull, Oltra y Ramón, 2017).

(cc) EY-NC-ND 2018, Universitat Politècnica de València 
b) Comparar los resultados en dos asignaturas diferentes y de titulaciones también distintas, con el fin de conocer el grado de comprensión de las dos competencias que son objeto de innovación docente por parte de los propios estudiantes.

c) Determinar si el alumnado responde positivamente a la utilización de audiovisuales para poder evaluar las competencias.

d) Establecer si el audiovisual utilizado sirve al alumnado para ayudarle a ver la aplicación de las competencias transversales.

\section{La utilización del audiovisual Tutankamon como herramienta para evaluar las competencias transversales en asignaturas punto de control}

\subsection{El pensamiento crítico. Breves apuntes}

El pensamiento crítico es parte del proceso para la resolución de problemas, enmarcado en un entorno de aprendizaje que requiere de alfabetización informacional (ALA, 2004). Algunas veces los términos "pensamiento crítico" y "conocimiento de la información” se usan conjuntamente sin una diferenciación clara (Bryan, 2014). Es importante que en el currículo del alumnado de educación superior se fomenten prácticas interdisciplinarias y creativas que ayuden a pensar acerca de las interacciones entre el hombre y el medio que le rodea, viéndolo desde una perspectiva de lograr un futuro más sostenible. Nuevos enfoques educativos servirán para facilitar un pensamiento interdisciplinario genuino, conducente al pensamiento crítico y la capacidad reflexiva (Howlett, 2016).

El pensamiento crítico o la reflexión crítica sugiere abrir esta acción para incorporar en el proceso cognitivo aspectos sociopolíticos, un cuestionamiento de los propios valores y suposiciones, con la finalidad de generar conocimiento desde un contexto más amplio. Por ello la reflexión crítica es relevante para la educación de adultos, y particularmente la educación superior. Como señala Brookfield (1995), un pensamiento crítico es el proceso mediante el cual los adultos cuestionan y luego reemplazan o replantean una suposición que, hasta ese momento, se ha aceptado acríticamente como la sabiduría del sentido común, así como el proceso a través del cual los adultos toman perspectivas alternativas sobre ideas, acciones, formas de razonamiento e ideologías previamente dadas por hechas. El pensamiento crítico y la práctica reflexiva han ganado en los últimos años cada vez más validez en el aprendizaje y en la educación superior, en lugar del trabajo de estudio en general, particularmente en los campos de desarrollo profesional, administrativo y, también, en el de la gestión en las organizaciones (Rigg, 2008).

Es difícil llegar a una conclusión precisa sobre qué competencias se requieren para ser un profesional emprendedor, y los estudios sobre este tema coinciden en que las competencias profesionales son a menudo psicológicas o sociales, más que habilidades específicas limitadas a la rama empresarial y, sin embargo, esto no suele constar en el currículo académico. Ello ha condicionado que la educación académica superior se centre profundamente en la transmisión del conocimiento académico ignorando ayudar a los estudiantes al crecimiento de carácter psicológico o sociológico (Taatila, 2010). Sánchez (2013) define las competencias como un conjunto de conocimientos, rasgos, actitudes y habilidades relacionadas que afectan a una parte del trabajo. Las universidades están 
cambiando el enfoque del currículo de sus alumnos, especialmente en aquellos que tienen una orientación empresarial, implementando estudios centrados en estas competencias empresariales, como la creatividad, la innovación, la autoeficiencia y la orientación hacia las acciones para ser más emprendedores (Gibson, 2018). En la actividad de la gestión cultural, tanto los profesionales como las empresas del ramo, deben abordar muchas acciones diferentes para alcanzar la eficiencia, desde el conocimiento de la normativa jurídica que le compete, pasando por la gestión de proyectos, el dominio del patrimonio cultural, la sociología del público o gestión económica y presupuestaria. Para Reinstein y Bayou (1997) la educación contable - imprescindible para la gestión económica y presupuestaria - debe proporcionar a los estudiantes el conjunto de habilidades requeridas que buscan los futuros empleadores, incluida la comunicación sólida, el análisis cuantitativo y las habilidades interpersonales e intelectuales.

En el entorno educativo se han utilizado series y películas de larga duración para ayudar tanto a desarrollar esta competencia como a que los estudiantes la perciban. La organización de estas actividases se encuentran relacionadas, muchas veces, con ciertas figuras cinematográficas, especialmente en el mundo de la arqueología y los museos. Narraciones tipo Hollywood donde los arqueólogos son individuos románticos y aventureros que presentan una visión distorsionada de los motivos del arqueólogo científico, al ponerlo al servicio de agencias gubernamentales ficticias o de personas muy ricas con la misión de salvar al mundo (Argyropoulos, 2014).

\subsection{La responsabilidad ética, medioambiental y profesional. Breves apuntes}

En el caso de esta competencia se trata de que el alumnado detecte elementos de acuerdo con lo que se considera como ética, buenas prácticas en el ámbito medioambiental y dentro de su profesión de futuro.

Por ese motivo, esta competencia queda fuertamente delimitada por la titulación analizada. Por ejemplo, en el caso del Máster en Conservación y Restauración de Bienes Culturales el propósito de la actividad era que el alumnado considerase que el futuro conservador o restaurador podía realizar sus actividades conforme a las prácticas adecuadas para no dañar una obra, no aplicar técnicas que implicaran una contaminación en el medio ambiente, así como que utilizara herramientas idóneas para lograr conservar y restaurar una obra de arte.

\section{Metodología: desarrollo de la innovación en el Máster de Gestión Cultural y en el Máster en Conservación y Restauración de Bienes Culturales de la Universitat Politècnica de València}

Vamos en este punto a exponer el desarrollo de la innovación que se ha llevado a cabo en dos titulaciones de la Universitat Politècnica de València.

A) En el Máster Universitario de Gestión Cultural. Se trata de un título interuniversitario entre la Universitat de València y la Universitat Politècnica de València. Se imparte la asignatura troncal "Marco jurídico aplicado a la protección del patrimonio y a los museos". La finalidad de esta asignatura es que el alumno adquiera conocimientos sobre la aplicación de un marco jurídico exigido a las administraciones públicas y a los ciudadanos en general,

(c) EY-NC-ND 2018, Universitat Politècnica de València

Congreso IN-RED (2018) 
para la protección del patrimonio cultural y la gestión de los museos. En cuanto a los contenidos se enseñan las principales normas jurídicas sobre protección del patrimonio histórico y cultural emitidas por la UNESCO, la Unión Europea, el estado español y la Comunidad Valenciana.

Entre las competencias básicas que se deben impulsar en la asignatura constan:

- Que los estudiantes sepan aplicar los conocimientos adquiridos y su capacidad de resolución de problemas en entornos nuevos o poco conocidos dentro de contextos más amplios (o multidisciplinares) relacionados con su área de estudio.

- Que los estudiantes sean capaces de integrar conocimientos y enfrentarse a la complejidad de formular juicios a partir de una información que, siendo incompleta o limitada, incluya reflexiones sobre las responsabilidades sociales y éticas vinculadas a la aplicación de sus conocimientos y juicios.

Además, esta asignatura tiene asignada como punto de control el desarrollo y evaluación de la competencia transversal "Pensamiento Crítico". Para trabajar dicha competencia transversal se realizaron dos actividades,

B) En el Master en Conservación y Restauración de Bienes Culturales. Se trata de un título impartido en la Universitat Politècnica de València. Dentro del mismo se imparte la asignatura de "Legislación del patrimonio cultural y creación de pequeñas empresas". Se estudian como contenidos principales la legislación de protección del patrimonio cultural tanto nacional como autonómico, así como los instrumentos normativos destinados al emprendedurismo.

Entre las competencias que se impulsan en esta asignatura, se encuentan:

-Trabajar y liderar equipos de forma efectiva para la consecución de objetivos comunes contribuyendo al desarrollo personal y profesional de los mismos en el ámbito de la Conservación y Restauración de Bienes Culturales.

-Capacidad para gestionar el patrimonio histórico-artístico, determinando las actuaciones necesarias para su conservación y disfrute.

En esta asignatura se trabaja la competencia de responsabilidad ética, medioambiental y profesional.

En ambos títulos para trabajar las competencias transversales indicadas (Pensamiento crítico y responsabilidad ética, medioambiental y profesional) se realizaron dos actividades una relacionada con el estudio y comentarios de un caso, y otra relacionada con un debate a partir de la visualización de un fragmento de una serie relacionada con el descubrimiento de un tesoro y su protección jurídica.

En lo que respecta a la actividad llevada a cabo, se trataba de utilizar el caso práctico basado en la serie de ficción núm. 12 del manual Casos Prácticos basados en series de ficción, referente a Tutankamon (Cabedo, Casar, Giménez, Lull, Oltra y Ramón, 2017). Esta serie trata sobre la temática del tesoro y la regulación del mismo, así como la gestión y su propiedad, en relación con el Código Civil, principalmente el artículo 352 que especifica 
que "se entiende por tesoro, para los efectos de la ley, el depósito oculto e ignorado de dinero, alhajas u otros objetos preciosos, cuya legítima pertenencia no conste.”.

En el minuto 14 del tercer episodio de dicha serie, Howard Carter comenta a la hija de Lord Carnarvon, Lady Evelyn, el sistema de reparto de beneficios del descubrimiento según los términos de la concesión: la mitad es para el estado egipcio y la otra mitad para Lord Carnarvon.

Tras el descubrimiento del tesoro en la antecámara funeraria el reto de Carter es la gestión y conservación de las piezas. Para ello solicita ayuda externa al Museo Metropolitano de Nueva York. Sin embargo, a los pocos días, las autoridades de El Cairo deciden cambiar la legislación de forma apresurada (hecho que les afectará, pues contempla el carácter retroactivo), por la cual, cualquier descubrimiento pertenece al estado de Egipto.

\section{Resultados obtenidos en el Máster de Gestión Cultural y en el Máster en Conservación y Restauración de Bienes Culturales respecto de las competencias analizadas}

Tras la revisión de la normativa jurídica actual que afecta a la protección del patrimonio en nuestro ordenamiento jurídico, se proyecta a los alumnos un fragmento del tercer capítulo de esta miniserie. Posteriormente se inicia un debate en el aula, donde los alumnos reflexionan sobre dos épocas completamente diferentes, dando sus opiniones sobre la legalidad o no de que los extranjeros se puedan apropiar de tesoros y patrimonio artístico de otros países económicamente desprotegidos, si las leyes pueden ser retroactivas, etc.

El debate sobre la miniserie sirvió para estimular la reflexión de los alumnos a partir del conocimiento del marco jurídico de protección al patrimonio, descubriendo que es habitual realizar reflexiones del pasado con esquemas de valores actuales, que pueden dificultar la comprensión de actividades y normas jurídicas enmarcadas en una sociedad diferente.

Concluida la actividad en el aula se pasó un breve test anónimo a los alumnos asistentes para valorar el grado de satisfacción y conocer opiniones sobre la actividad. Participaron trece alumnos. Las preguntas planteadas se debían de responder con una escala de valoración de 1 a 5, siendo el uno un total desacuerdo y el cinco un total acuerdo.

Las preguntas que se les formularon fueron las siguientes:

Primera pregunta: ¿Es interesante el empleo en el aula de elementos audiovisuales para debatir el problema de casos prácticos?

Segunda pregunta: ¿Habéis detectado elementos jurídicos en el vídeo?

Tercera pregunta: ¿La actividad realizada, visualización del vídeo más el debate en clase, os ha ayudado a comprender mejor los conocimientos de la asignatura?

Cuarta pregunta: ¿El vídeo os ha resultado útil para el desarrollo de la competencia transversal de "Pensamiento crítico"?

Quinta pregunta: ¿La actividad os ha supuesto un estímulo para valorar la importancia del marco jurídico en la protección del patrimonio cultural?

(cc) EY-NC-ND 2018, Universitat Politècnica de València

Congreso IN-RED (2018) 
Los resultados en el Máster de Gestión Cultural fueron los siguientes:

a) A la primera pregunta, un 69\% están totalmente de acuerdo y un 31 \% están de acuerdo, lo que indica que consideran positivo el empleo de audiovisuales en la docencia (Figura 1).

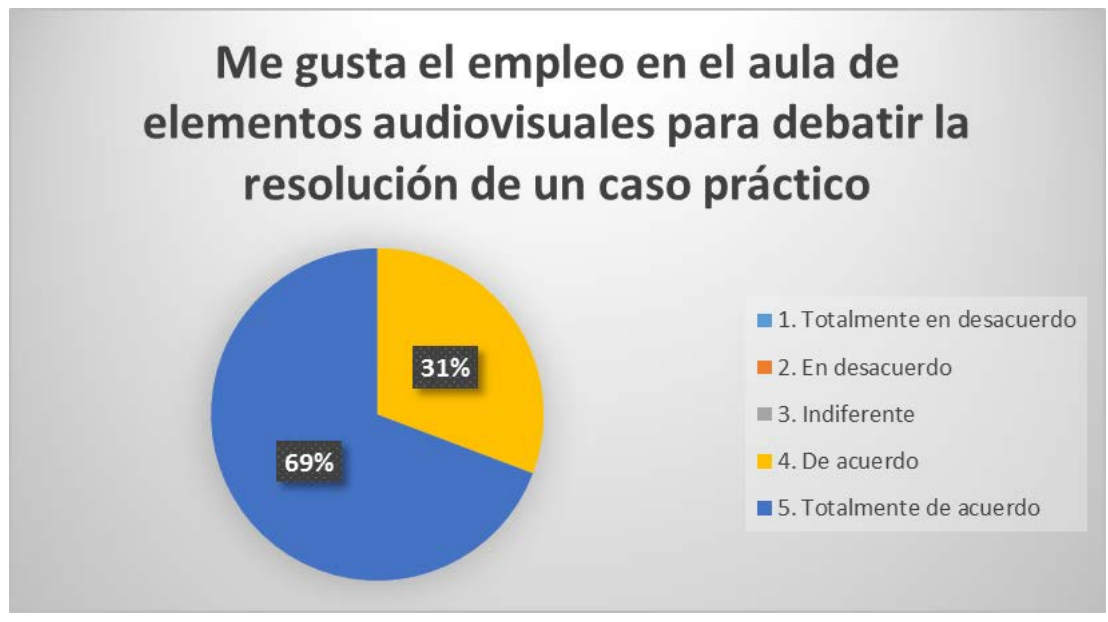

Fig. 1. Porcentaje respecto a la pregunta de "Me gusta el empleo en el aula de elementos audiovisuales para debater la resolución de un caso práctico. Fuente: elaboración propia.

b) A la segunda pregunta, un $16 \%$ no lo detectó, pero el $84 \%$ sí que ha valorado de alguna forma que en el vídeo se mencionaban elementos jurídicos (Figura 2). Se planteaba un cambio de la normaiva jurídica en Egipto a raíz del descubrimiento de la tumba de Tutankamón.

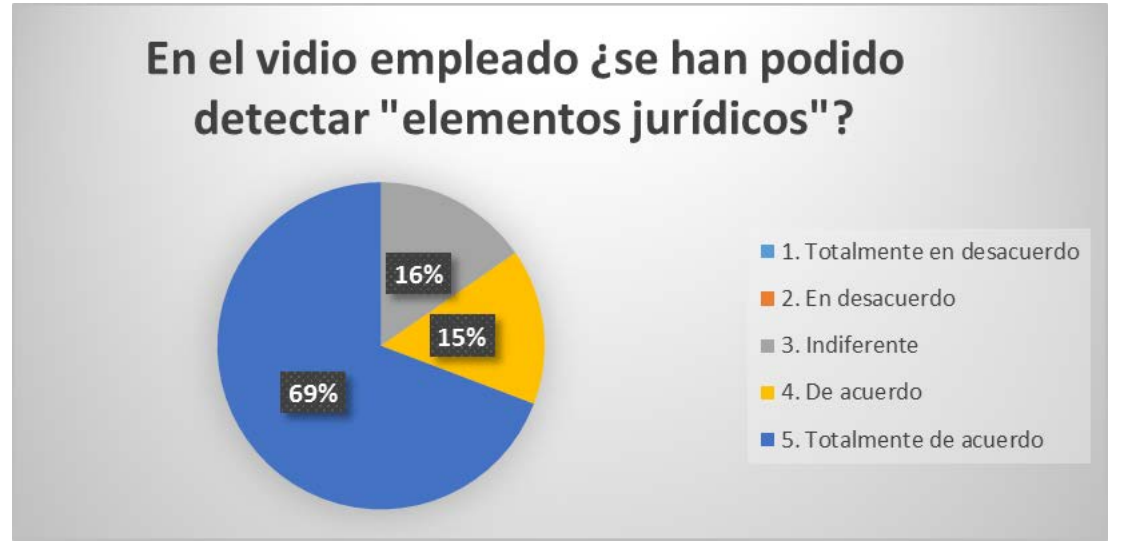

Fig. 2. Porcentaje respecto a la pregunta de "En el vídeo empleado ¿̇e han podido detectar «elementos jurídicos»”. Fuente: elaboración propia.

c) A la tercera pregunta, en general, un $85 \%$ lo considera positivo (Figura 3). 


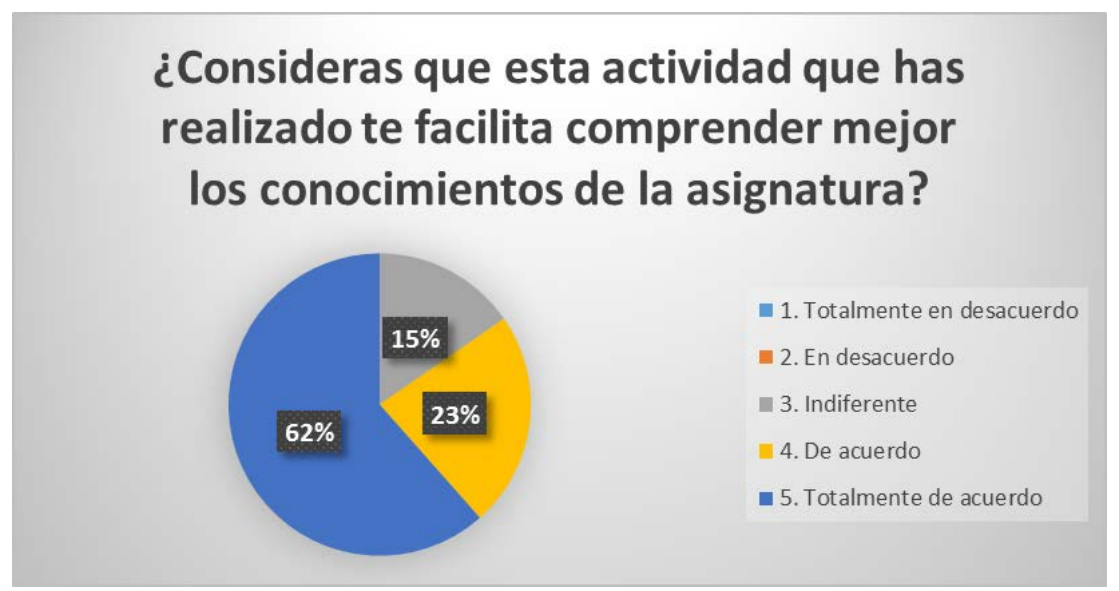

Fig. 3. Porcentaje respecto a la pregunta de “¿Consideras que esta actividad que has realizado te facilita comprender mejor los conocimientos de la asignatura?”. Fuente: elaboración propia.

d) A la cuarta pregunta, un 92\% mostró estár de acuerdo o totalmente de acuerdo, mientras que un $8 \%$ consideró indiferente la relación de este vídeo con el desarrollo de la competencia (Figura 4).

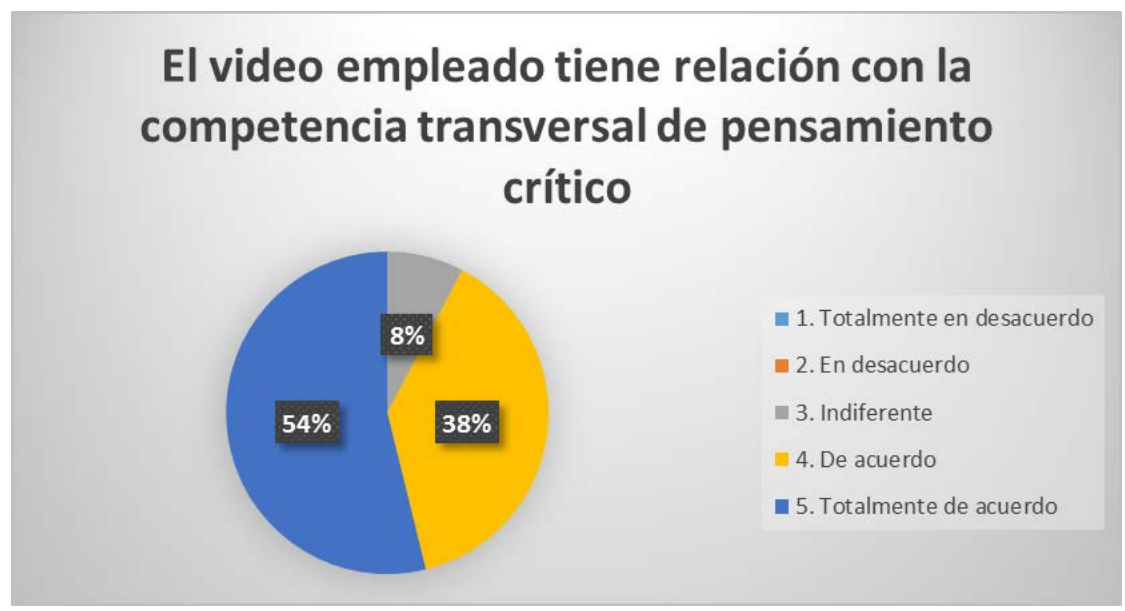

Fig. 4. Porcentaje respecto a la pregunta de "El video empleado tiene relación con la competencia transversal de pensamiento crítico”. Fuente: elaboración propia.

e) A la quinta pregunta, la totalidad de los alumnos estuvieron de acuerdo o muy de acuerdo (Figura 5). 


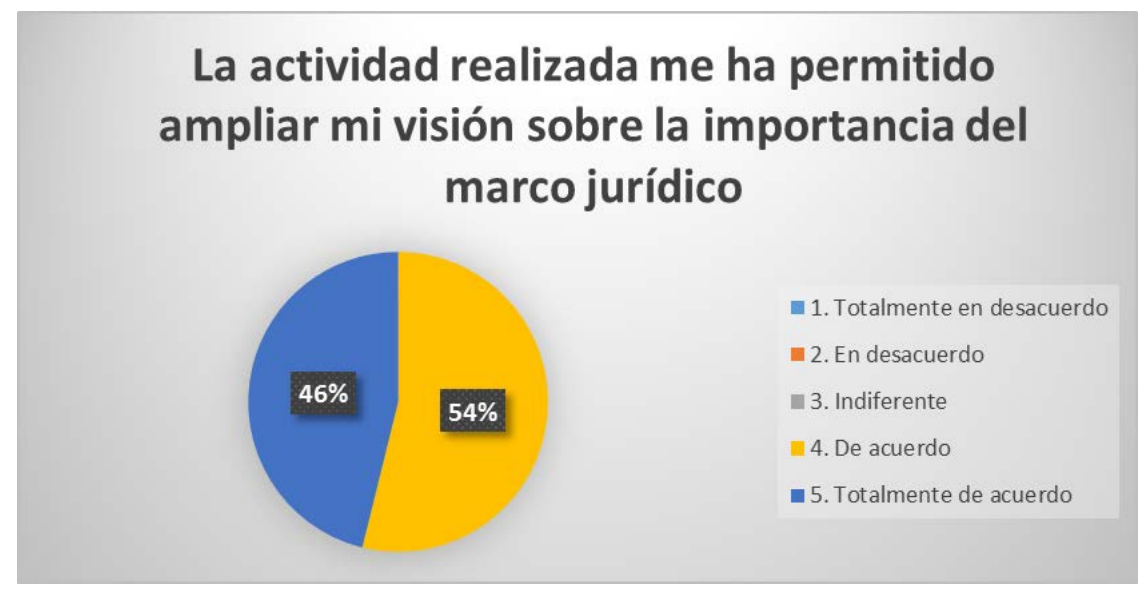

Fig. 5. Porcentaje respecto a la pregunta de "La actividad realizada me ha permitido ampliar mi visión sobre la importancia del marco jurídico”. Fuente: elaboración propia.

Por último, se les dejó a los alumnos una pregunta abierta para que diesen su opinión sobre cómo mejorar la actividad. Hubo algunas sugerencias, como poner audiovisuales de casos reales más recientes para detectar mejor las similitudes con la normativa jurídica actual; realizar grupos en el aula para posteriormente debatir en común, como forma de ampliar la participación a todos los alumnos (de lo contrario, unas cuantas personas tienen más protagonismo); algunos alumnos han coincidido en que les parece muy útil pero que ha faltado tiempo para debatir más en el aula, pudiéndose ampliar también a visualizar películas, videoclips, programas de televisión, etc.

Se formulo respecto al Máster en Conservación y Restauración de Bienes Culturales una única pregunta enfocada a detectar si el alumnado podía relacionar el episodio con la competencia transversal de responsabilidad ética, medio ambiental y profesional, y se le preguntó:

Pregunta única: ¿Puedes aplicar la competencia transversal de responsabilidad ética, medio ambiental y profesional en el episodio de Tutankamón?

Los resultados en el Máster en Conservación y Restauración de Bienes Culturales fueron los siguientes:

Se pasó la encuesta a un total de 30 alumnos de los que contestaron el 76.7\% (23 personas).

A la pregunta sobre si aplicarían la competencia transversal de responsabilidad ética, medio ambiental y profesional en el episodio de Tuankamón, un total de 11 contestaron afirmativamente que podrían aplicar la competencia transversal en la actividad que habían realizado, mientras que los 12 restantes indicaron, por el contrario, que no la podían aplicar. Tal y como se puede apreciar en la Figura 6, el porcentaje obtenido refleja una polaridad: prácticamente la mitad de los alumnos consideraba que sí podía aplicarse y la otra mitad que no. 


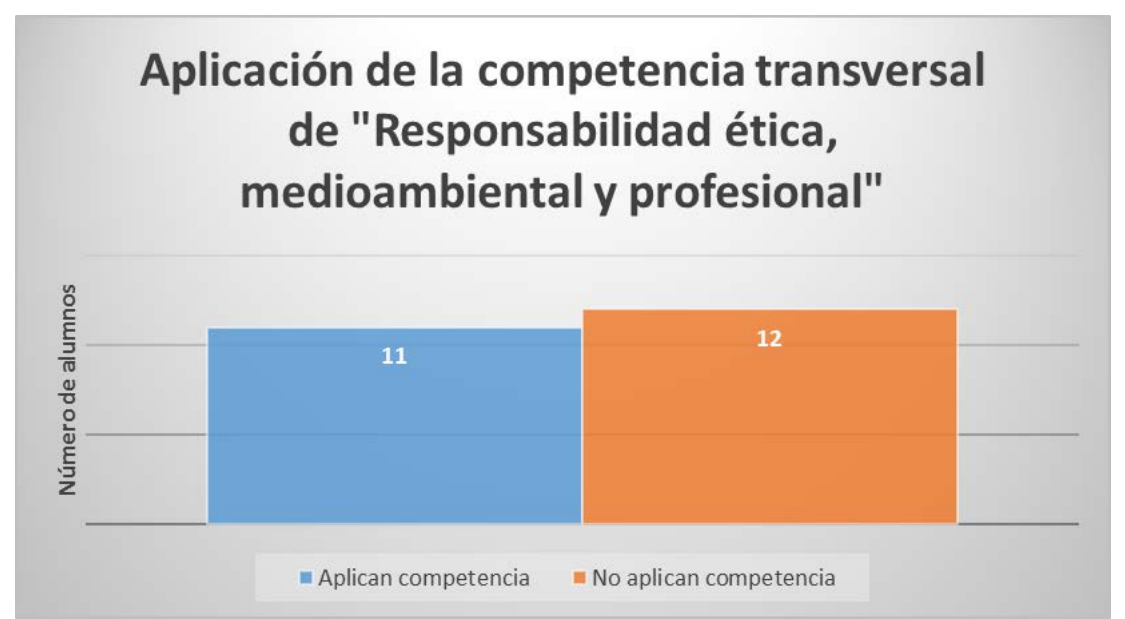

Fig. 6. Número de alumnos que consideraban que se podía aplicar la competencia transversal de "Responsabilidad ética, medioambiental y profesional" en el audiovisual Tutankamon. Fuente: elaboración propia

\section{Conclusiones}

La competencia transversal de "Pensamiento Crítico" se puede desarrollar en el aula como forma de aprendizaje en el proceso cognitivo. La actividad de visualizar un fragmento de una serie y un posterior debate permite que los alumnos participen activamente dando sus opiniones con el fundamento de los contenidos aprendidos en clase, y con sus conocimientos socioculturales actuales. La valoración por parte de los alumnos ha sido muy positiva, y les ha permitido ampliar la visión de la importancia del marco jurídico en la gestión del patrimonio cultural.

Respecto a la competencia transversal de responsabilidad ética, medioambiental y profesional, hemos observado que está muy equilibrado el número de alumnos que detectaba la competencia en el caso del audiovisual Tutankamon y los que consideraban que no se aplicaba.

Hemos podido constatar que las preguntas abiertas resultan más completas y aportan más información que las de tipo test o cerradas, aunque el inconveniente es precisamente el número de alumnos, siendo más viable en los grupos pequeños las preguntas abiertas y para los grupos más amplios las abiertas o explicativas, por distintas razones, entre ellas, la corrección de las actividades para poderlas evaluar.

También denota la influencia el alumnado respecto a su posibilidad de eplicar la percepción del episodio, que favorece a los que tienen más aptitudes de redacción, que las preguntas cerradas, que precisan de una mayor atención por parte del discente.

Concluimos que la utilización de un episodio audivisual, en este caso, de la serie de Tutankamón nos ha permitido evaluar dos diferentes competencias transversales que pretendíamos detectaran el alumnado, de tal forma que ello nos permite considerar que la experiencia de la utilización de casos prácticos basados en series es adecuado como método evaluatorio de distintas competencias. Y que aunque sea utilizado el mismo caso por 
diferentes docentes y en títulos distintos, los resultados nos aproximan a la pretensión de la utilización de este medio para permitir valorar el grado de aprendizaje de la competencia objeto de estudio.

\section{Agradecimientos}

Trabajo realizado en el marco del Proyecto I+D Excelencia del Ministerio de Economía y Competitividad (DER2015-65810-P), 2016-2018; Proyecto "Derecho civil valenciano y europeo" del Programa Prometeo para Grupos de Investigación de Excelencia de la Conselleria de Educación, Cultura y Deporte, GVPROMETEOII2015-014 y Proyecto de Innovación y Mejora Educativa (PIMEs) "La utilización de los documentales como herramienta para la evaluación de la competencia transversal CT07 Responsabilidad ética, medioambiental y profesional”, en la convocatoria llevada a cabo en la Universitat Politècnica para el curso 2017-2018, Vicerrectorado de Estudios y Convergencia Europea de la Universitat Politècnica de València. Investigadora principal: Francisca Ramón Fernández. Investigadores: Vicente Cabedo Mallol, María Emilia Casar Furió, Vicent Giménez Chornet, Cristina Lull Noguera y Juan Vicente Oltra Gutiérrez.

\section{Referencias}

ALA (AMERICAN LiBrary Association) (2004). Information Literacy Competency Standards for Higher Education .

$<\underline{\text { http://www.ala.org/Template.cfm?Section=Home\&template=\%2FContentManagement\%2 }}$ FContentDisplay.cfm\&ContentID=33553> [Consulta: 20 de febrero de 2018].

ARgYropoulos, V. et al. (2014). "Museum Education and Archaeological Ethics: An Approach to the Illicit Trade of Antiquities" en Journal of Conservation and Museum Studies, 12(1), p. 2.

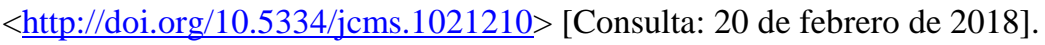

BROOKFIELD, S. (1995). “Adult learning: An overview” en International encyclopedia of education, vol. 10, p. 375-380.

$<\underline{\text { http://citeseerx.ist.psu.edu/viewdoc/download?doi=10.1.1.152.4176\&rep=rep1\&type=pdf }}$ $>$ [Consulta: 19 de febrero de 2018].

BRYAN, J. E. (2014) "Critical thinking, information literacy and quality enhancement plans” en Reference Services Review, vol. 42, issue: 3, p. 388-402.

$<$ https://doi.org/10.1108/RSR-01-2014-0001> [Consulta: 20 de febrero de 2018].

CABEDO MALLOL, V., CASAR FURIÓ, M. E., GIMÉNEZ CHORNET, V., LULL NOGUERA, C., OLTRA GUTIÉRREZ, J. V. y RAMÓN FERNÁNDEZ, F. (2017). Casos prácticos jurídicos basados en series de ficción. Francisca Ramón Fernández (coord.). Valencia: Tirant lo Blanch.

GiBSON, D., TAVLARIDIS, V. (2018) "Work-based learning for enterprise education? The case of Liverpool John Moores University "live" civic engagement projects for

(c) ) EY-NC-ND 2018, Universitat Politècnica de València 
students" en Higher Education, Skills and Work-Based Learning, vol. 8, issue 1, p.5-14.

<https://doi.org/10.1108/HESWBL-12-2017-0100> [Consulta: 21 de febrero de 2018].

HOWLETT, C.; FERREIRA, J. A.; BlOMFIELD, J. (2016) “Teaching sustainable development in higher education: Building critical, reflective thinkers through an interdisciplinary approach” en International Journal of Sustainability in Higher Education, vol. 17, issue 3, p. 305-321.

$<$ https://doi.org/10.1108/IJSHE-07-2014-0102> [Consulta: 21 de febrero de 2018].

KOVACSICS, v. (2015). The good wife: de cordero a lobo, en cinco temporadas. Caimán cuadernos de cine, núm. 35, pág. 13.

REINSTEIN, A.; BAYOU, M. E. (1997) "Critical thinking in accounting education: processes, skills and applications" en Managerial Auditing Journal, vol. 12, issue 7, p.336342.

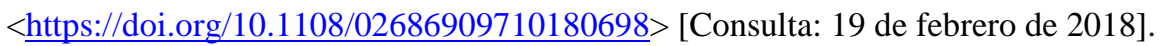

RIGG, C.; TREHAN, K.. (2008) "Critical reflection in the workplace: is it just too difficult?" en Journal of European Industrial Training, vol. 32, issue 5, p.374-384.

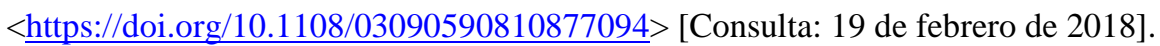

SÁNCHEZ, J. C. (2013). "El impacto de un programa de educación empresarial en las competencias e intenciones empresariales" en Journal of Small Business Management, 51 (3), 447-465.

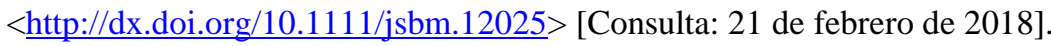

TAATILA, V. P. (2010) "Learning entrepreneurship in higher education" en Education + Training, vol. 52, issue 1, p.48-61.

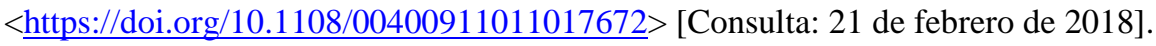

UPV. (2016). Papel de las rúbricas en la evaluación de las competencias transversales UPV.

Disponible en: <http://www.upv.es/entidades/ICE/info/U0702453.pdf> [Consulta: 25 de febrero de 2018].

VILLA, A. Y POBLETE, M. (2007). Aprendizaje basado en competencias. Una propuesta para la evaluación de las competencias genéricas. Bilbao: Ediciones Mensajero.

(cc) EY-NC-ND 2018, Universitat Politècnica de València

Congreso IN-RED (2018) 